\title{
ON INTEGRAL FUNCTIONS OF INTEGRAL OR ZERO ORDER
}

\author{
S. M. SHAH
}

Let $F(z)$ be an integral function of finite order $\rho$. We write $F(z)=z^{k} e^{g(z)} f(z)$ where $g(z)$ is a polynomial of degree $q \leqq \rho$ and

$$
f(z)=\prod_{1}^{\infty}\left\{\left(1-\frac{z}{a_{n}}\right) \exp \left(\frac{z}{a_{n}}+\cdots+\frac{1}{p}\left(\frac{z}{a_{n}}\right)^{p}\right)\right\}
$$

is the canonical product of order $\rho_{1}$ and genus $p$. Let $M(r, F)$ $=\max _{|z|=r}|F(z)|$ and $n(r, F-a)=n(r, a)$ be the number of zeros of $F(z)-a$ in $|z|=r$. In an earlier paper ${ }^{1}$ I proved the following result.

THEOREM 1. If $F(z)$ be of integral order $\rho$ and if the genus of the canonical product $f(z)$ be $p=\rho$, then

$$
\liminf _{r=\infty} \frac{\log M(r, F)}{n(r, F) \phi(r)}=0
$$

where $\phi(x)$ is any positive continuous increasing function of the real variable $x$ such that

$$
\int_{a}^{\infty} \frac{d x}{x \phi(x)}
$$

is convergent.

In this note I prove a similar result for the canonical products of order $\rho$ and genus $p=\rho-1$, and discuss whether the result can be extended to integral functions which are not canonical products. The main result is the following.

THEOREM 2. If $f(z)$ is a canonical product of integral order $\rho$ and genus $p=\rho-1$ then

$$
\liminf _{r=\infty} \frac{\log M(r, f)}{n(r, f) \Phi(r)}=0
$$

where $\Phi(x)$ is any positive increasing function such that

$$
\int_{a}^{\infty} \frac{d x}{x \Phi(x)}
$$

Received by the editors July 20,1941 .

${ }^{1} A$ Theorem on integral functions of integral order, Journal of the London Mathematical Society, vol. 15 (1940), pp. 23-31. I shall refer to this paper as (1). 
is convergent and

$$
\Phi(x) / x^{\alpha}
$$

is monotonic for all large $x$, say $x \geqq \Delta>0 ; \alpha$ a constant such that $0<\alpha<1$.

LEMMA 1. For all $r \geqq r_{0}(A, \beta)$

$$
J=\int_{\Delta_{1}}^{r} \frac{d x}{x^{\beta} \Phi(x)}<\frac{A r^{1-\beta}}{\log r}
$$

where $A$ and $\Delta_{1}$ are positive constants, and $\beta$ is a constant such that $0<\beta<1$.

Proof. From the convergence of the integral in (4), we have $\log x<\Phi(x)$ for all $x \geqq \Delta_{2}$. Hence for $r \geqq r_{0}$

$$
J=\int_{\Delta_{1}}^{r^{1 / 2}}+\int_{r^{1 / 2}}^{r} \leqq \frac{1}{\Phi\left(\Delta_{1}\right)} \frac{r^{(1-\beta) / 2}}{(1-\beta)}+\frac{2}{(1-\beta)} \frac{r^{1-\beta}}{\log r}<\frac{A r^{1-\beta}}{\log r} .
$$

LEMMA 2. Suppose that the real functions $\psi(x)$ and $\theta(x)$ satisfy the following conditions:

(1) $\psi(x)$ is continuous in $(\delta, \infty)$ where $\delta>0$, except for isolated points where $\psi(x)$ has ordinary left-hand discontinuities.

(2) $\psi(x)$ is non-increasing as $x \geqq \delta$ increases in any interval between two consecutive discontinuities.

(3) $\theta(x)$ is a positive continuous increasing function for $x \geqq \delta$.

$$
\limsup _{x=\infty} \psi(x)=\infty, \quad \lim _{x=\infty} \sup \frac{\psi(x)}{\theta(x)}=0 .
$$

Then we can find a sequence $\left\{x_{n}\right\}$ of values of $x$ tending to $\infty$ such that the two inequalities

are satisfied simultaneously.

$$
\begin{array}{lr}
\psi(x) \leqq \psi\left(x_{n}\right), & x_{1} \leqq x<x_{n}, \\
\frac{\psi(x)}{\theta(x)} \leqq \frac{\psi\left(x_{n}\right)}{\theta\left(x_{n}\right)}, & x>x_{n},
\end{array}
$$

The $x_{n}$ are points of discontinuity so that $\psi\left(x_{n}\right)=\psi\left(x_{n}+0\right)$ and $x_{1}$ is the first point of discontinuity in $(\delta, \infty)$.

The proof is similar to that of Lemma 2 of my paper referred to above, and is based on the following lemma of Pólya. ${ }^{2}$

If

$$
\begin{array}{lr}
l_{1}, l_{2}, l_{3}, \cdots, & l_{m}>0, \\
s_{1}, s_{2}, s_{3}, \cdots, & s_{1}>0 ; s_{m+1}>s_{m} ; m=1,2,3, \cdots,
\end{array}
$$

${ }^{2}$ Mathematische Annalen, vol. 88 (1923), p. 170. 
are two sequences of positive numbers, of which the second is monotonic and increasing, such that

$$
\lim _{m=\infty} l_{m}=0, \quad \quad \limsup _{m=\infty} l_{m} s_{m}=\infty,
$$

then we can find an infinite sequence $\{n\}$ of the indices $n$ such that the two sets of inequalities

$$
\begin{aligned}
l_{n} & >l_{\nu}, & & \nu>n, \\
l_{n} s_{n} & >l_{\mu} s_{\mu}, & & \mu<n,
\end{aligned}
$$

are satisfied simultaneously.

To prove Theorem 2 we first consider the case when

$$
\limsup _{r=\infty} \frac{n(r, f) \Phi(r)}{r^{p+1}}>0 .
$$

We have

$$
r^{p+1}<A n(r) \Phi(r)
$$

for an infinity of values $r=R_{n}$ tending to $\infty$ and so

$$
\frac{\log M\left(R_{n}\right)}{n\left(R_{n}\right) \Phi\left(R_{n}\right)}<\frac{A \log M\left(R_{n}\right)}{R_{n}^{p+1}} \rightarrow 0 \quad \text { as } n \rightarrow \infty \text {. }
$$

Hence

$$
\liminf _{r=\infty} \frac{\log M(r)}{n(r) \Phi(r)}=0
$$

Suppose secondly that

$$
\lim _{r=\infty} \frac{n(r) \Phi(r)}{r^{p+1}}=0
$$

Here $\Phi(x) / x^{\alpha}$ must be monotonic decreasing, for if not $\Phi(x) \geqq A x^{\alpha}$, and so

$$
\limsup _{r=\infty} \frac{n(r) \Phi(r)}{r^{p+1}} \geqq A \lim \sup _{r=\infty} \frac{n(r)}{r^{p+1-\alpha}}=\infty,
$$

contradicting hypothesis (5.2); so $\Phi(x) / x^{\alpha}$ is monotonic decreasing for $x \geqq \Delta$. We apply Lemma 2 putting

$$
\psi(x)=\frac{n(x) \Phi(x)}{x^{p+1-\beta}}=n(x) \frac{\Phi(x)}{x^{\alpha}} \frac{1}{x^{p+1-\alpha-\beta}},
$$

and choosing $\theta(x)=x^{\beta}, \beta$ a constant such that $0<\beta<1-\alpha, \delta=\Delta$. The 
conditions of Lemma 2 are satisfied, and hence, putting $x_{n}=R$ we obtain

$$
\begin{array}{ll}
\frac{n(x) \Phi(x)}{x^{p+1-\beta}} \leqq \frac{n(R) \Phi(R)}{R^{p+1-\beta}}, & \text { for } \Delta \leqq x_{1} \leqq x \leqq R, \\
\frac{n(x) \Phi(x)}{x^{p+1}} \leqq \frac{n(R) \Phi(R)}{R^{p+1}}, & \text { for } x>R .
\end{array}
$$

Thus for $R>x_{1}$,

$\log M(R, f)<A I(R, f)$

$$
\begin{aligned}
& =A \int_{0}^{\infty} \frac{n(x, f)}{x^{p+1}} \frac{R^{p+1}}{(x+R)} d x \\
& \leqq A\left\{A_{1} R^{p} \int_{x_{1}}^{R} \frac{n(x)}{x^{p+1}} d x+R^{p+1} \int_{R}^{\infty} \frac{n(x)}{x^{p+2}} d x\right\} \\
& \leqq A\left\{A_{1} R^{p} \frac{n(R) \Phi(R)}{R^{p+1-\beta}} \int_{x_{1}}^{R} \frac{d x}{x^{\beta} \Phi(x)}+n(R) \Phi(R) \int_{R}^{\infty} \frac{d x}{x \Phi(x)}\right\} \\
& \leqq A\left\{\frac{A_{2} n(R) \Phi(R)}{\log R}+o(n(R) \Phi(R))\right\} .
\end{aligned}
$$

Hence

$$
\lim _{r=\infty} \inf \frac{\log M(r, f)}{n(r, f) \Phi(r)} \leqq A \liminf _{r=\infty} \frac{I(r, f)}{n(r, f) \Phi(r)}=0
$$

and this completes the proof of the theorem.

COROLLARY. If $F(z)=z^{k} e^{g(z)} f(z)$ is of integral order $\rho$ and genus $g=\rho-1$ then

$$
\lim _{r=\infty} \inf \frac{\log M(r, F)}{n(r, f) \Phi(r)}=0 .
$$

We have $g=\rho-1=\max (p, q)$. It is easily seen that $p=\rho-1$, $q \leqq \rho-1$ and

$$
\log M(r, F)<A\left\{r^{p-1}+\log r\right\}+\log M(r, f) .
$$

If

$$
\limsup _{r=\infty} \frac{n(r, f) \Phi(r)}{r^{p+1}}>0
$$

then $R_{n}$ being defined in (5.1), 


$$
\frac{\log M\left(R_{n}, F\right)}{n\left(R_{n}, F\right) \Phi\left(R_{n}\right)}<\frac{A\left\{R_{n}^{\rho-1}+\log R_{n}\right\}}{n\left(R_{n}, F\right) \Phi\left(R_{n}\right)}+\frac{\log M\left(R_{n}, f\right)}{n\left(R_{n}, f\right) \Phi\left(R_{n}\right)} .
$$

Hence

$$
\liminf _{r=\infty} \frac{\log M(r, F)}{n(r, F) \Phi(r)}=0
$$

If now

$$
\lim _{r=\infty} \frac{n(r, f) \Phi(r)}{r^{p+1}}=0
$$

then for all large $r$

$$
\log M(r, F)<A\left\{r^{\rho-1}+\log r\right\}+A I(r, f)<A_{3} I(r, f)
$$

and hence from (6) the required result follows.

The condition (4) on $\Phi(x)$ is sufficient but not necessary ${ }^{3}$ for (3) and (7) to hold. The condition (4.1) is also not necessary for we can take $\Phi(x)$ to be any function

$$
\left(l_{1} x\right)\left(l_{2} x\right) \cdots\left(l_{k-1} x\right)\left(l_{k} x\right)^{1+\eta}, \quad \eta>0,
$$

of the logarithmic comparison scale, and hence any function for which

$$
\liminf _{x=\infty} \frac{\Phi(x)}{\left(l_{1} x\right)\left(l_{2} x\right) \cdots\left(l_{k-1} x\right)\left(l_{k} x\right)^{1+\eta}} \geqq A>0 .
$$

We can take $\Phi(x)$ to be any positive $L$ function ${ }^{4}$ which satisfies (4) but we cannot take $\Phi(x)$ (or $\phi(x)$ in Theorem 1$)$ to be $\left(l_{1} x\right)\left(l_{2} x\right) \cdots\left(l_{k} x\right)$.

Consider for instance

$$
f_{1}(z)=\prod_{N}^{\infty}\left\{1-\frac{z}{a_{n}}\right\}, \quad f_{2}(z)=\prod_{N}^{\infty}\left\{\left(1-\frac{z}{a_{n}^{\prime}}\right) \exp \left(\frac{z}{a_{n}^{\prime}}\right)\right\},
$$

where

$$
\begin{aligned}
a_{n} & =-n\left(l_{1} n\right) \cdots\left(l_{k} n\right)\left(l_{k+1} n\right)^{2}, \\
a_{n}^{\prime} & =n\left(l_{1} n\right) \cdots\left(l_{k} n\right)\left(l_{k+1} n\right) .
\end{aligned}
$$

The functions $f_{1}(z)$ and $f_{2}(z)$ are canonical products of order 1 . The genus of $f_{1}(z)$ is 0 , and of $f_{2}(z)$ is 1 . For each of them we have

$$
\lim _{r=\infty} \frac{\log M(r)}{n(r)\left(l_{1} r\right) \cdots\left(l_{k} r\right)}=\infty .
$$

${ }^{3}$ Cf. p. 4 of (1).

${ }^{4}$ For definition see G. H. Hardy, Orders of Infinity, 1924, p. 17. 
In what follows we shall take $\phi(x)$ to be a positive $L$ function satisfying the condition (2).

Suppose now $F(z)$ is of integral order $\rho$. There are four possibilities:

(1) $\rho_{1}<\rho, p \leqq \rho_{1}, q=\rho$,

(2) $\rho_{1}=p=\rho, q \leqq \rho$,

(3) $\rho_{1}=q=\rho, p=\rho-1$,

(4) $\rho_{1}=\rho, q<\rho, p=\rho-1$.

Combining the above results we have in cases (2) and (4)

$$
\liminf _{r=\infty} \frac{\log M(r, F)}{n(r, F) \phi(r)}=0 .
$$

In cases (1) and (3), (9) does not hold. ${ }^{5}$ For functions of fractional order and zero order ${ }^{6}$ it certainly holds. In particular (9) is true for any canonical product of finite order; it also holds for functions of maximum or minimum type, order $\rho$.

It is known that if $F(z)$ is of integral order $\rho$, then ${ }^{7}$

$$
\lim _{r=\infty} \inf \frac{\log M(r, F)}{n(r, F-a)}<\infty
$$

for every $a$, except possibly a single exceptional value of $a$. Since $F(z)$ and $F(z)-a$ belong to the same type, we deduce from (9) that if $F(z)$ is of maximum or minimum type, order $\rho$, where $\rho$ is an integer, then

$$
\liminf _{r=\infty} \frac{\log M(r, F)}{n(r, F-a) \phi(r)}=0
$$

for every $a$. If $F(z)$ is of mean type then (11) need not hold for one exceptional value of $a$. For example, $z e^{z}$ and

$$
e^{z} \prod_{2}^{\infty}\left\{1+\frac{z}{n(\log n)^{2}}\right\}
$$

are both functions of mean type, order 1 . For each of these two functions

$$
\lim _{r=\infty} \frac{\log M(r, F)}{n(r, F-0)(\log r)^{3 / 2}}=\infty .
$$

MUSLIM UNIVERSITY

ALIGARH, INDia

s (1), p. 29.

(1), pp. 29-30.

${ }^{7}$ G. Valiron, Lectures on the General Theory of Integral Functions, 1923, p. 86. 